\title{
Transcriptome Characterization and Identification of Molecular Markers (SNP, SSR, and Indels) in the Medicinal Plant Sarcandra glabra spp.
}

\author{
Yanqin Xu $\mathbb{D},{ }^{1}$ Shuyun Tian $\mathbb{D}^{\mathbb{C}},{ }^{1}$ Renqing $\mathrm{Li}\left(\mathbb{D},{ }^{1}\right.$ Xiaofang Huang $\mathbb{D}^{1},{ }^{1}$ Fengqin $\mathrm{Li} \mathbb{D},{ }^{1}$ \\ Fei Ge ${ }^{\circ},{ }^{1}$ Wenzhen Huang $\left({ }^{1},{ }^{1}\right.$ and Yin Zhou $\oplus^{2,3}$ \\ ${ }^{1}$ College of Pharmacy, Jiangxi University of Traditional Chinese Medicine, Nanchang 330004, China \\ ${ }^{2}$ Center of Applied Biotechnology, Wuhan University of Bioengineering, Wuhan 430415, China \\ ${ }^{3}$ College of Bioscience and Biotechnology, Wuhan University of Bioengineering, Wuhan 430415, China \\ Correspondence should be addressed to Yin Zhou; ripplet0931@hotmail.com
}

Received 12 March 2021; Accepted 28 June 2021; Published 8 July 2021

Academic Editor: Biaolin Hu

Copyright (c) 2021 Yanqin Xu et al. This is an open access article distributed under the Creative Commons Attribution License, which permits unrestricted use, distribution, and reproduction in any medium, provided the original work is properly cited.

\begin{abstract}
Sarcandra glabra has significant metabolically active bioingredients of pharmaceutical importance. The deficiency of molecular markers for S. glabra is a hindrance in molecular breeding for genetic improvement. In this study, 57.756 million pair-end reads were generated by transcriptome sequencing in S. glabra (Thunb.) Nakai and its subspecies S. glabra ssp. brachystachys. A total of 141,954 unigenes with $646.63 \mathrm{bp}$ average length were assembled. A total of 25,620 simple sequence repeats, 726,476 single nucleotide polymorphisms, and 42,939 insertions and deletions were identified, and the associated unigenes and differentially expressed genes were characterized. This work enhanced the molecular marker resources and will facilitate molecular breeding and gene mining in S. glabra spp.
\end{abstract}

\section{Background}

Sarcandra glabra (Chloranthaceae family) is an evergreen, smooth shrub, and grows up to one-meter height. It is originated in tropical climate of south Asia. It especially grows in China, Japan, Philippines, Vietnam, Korea, Cambodia, Malaysia, India, Sri Lanka, and other areas including areas of North, Central, and South America and some areas of the Pacific [1]. It has been known for its medicinal values in the treatment of joint and cancer problems [2]. S. glabra extract (SGE) contains high amount of powerful antiinflammatory compounds including isofraxidin, terpenoid saponins, fumaric acid, caffeic acid, rosmarinic acid, and caffeoylquinic acid [2]. SGE is widely used in Traditional Chinese Medicines to help with arthritis, pain, swelling, and redness [3]. It may have multifarious medicinal values as anti-inflammatory [4], antitumor [5], anti-infection [1], and antioxidant [6] bioactivities as described in recent studies. SGE also has the ability to attenuate hyperglycemia [7], which could be useful to recycle the industrial wastes for beneficial by-products [2]. S. glabra seeds can be dried and roasted for human consumption. Besides, it can also be used as an in- or outdoor ornamental plant. Owing to its significant chemical, rheological, and pharmacological importance $[1,2]$, it is important to improve of $S$. glabra varieties with wider acceptance and enriched with bioactive components.

The marker-based breeding techniques have been widely adapted for plant improvement and genetic conservation [8]. The information about the availability of molecular markers for genetic mapping and map-based gene cloning is always a basic need for any breeding or genomic research. Among DNA markers, single nucleotide polymorphisms (SNPs), simple sequence repeats (SSRs), and insertions and deletions (InDels) are the most simple and valuable markers [9]. The microsatellites are tandem repeats of various mono-, di-, tri-, tetra-, penta-, and hexanucleotide sequence motifs of variable lengths [10], which have been identified and manipulated in various crops [11]. SSR markers have always been 
preferred for their user-friendly properties and for their random genome distribution, simplicity of use, high level of polymorphism, high clarity, low operational cost, reproducibility, hypervariability, ease of multiplexing, amenability to automation, and use with low quality DNA [11]. Recently, high-throughput sequencing methods have widely been adapted for rapid identification of SNP, SSR, and InDel markers, especially in nonmodel plants [12].

The next-generation sequencing of cDNA pool obtained from extracted RNA of plant tissues is generally known as RNA-seq. The RNA-seq or transcriptomics approach can be used to obtain a huge number of expressed sequences which can further be used to design molecular markers. RNA-seq-based SSRs, SNPs, and InDels are associated with protein coding genes and their relevant translated regions [13]. The transcriptome-based markers facilitate the understanding of their link with phenotypic variation and/or functional genes [14]. They are transferable among closely related species [13] because of their highly conserved domains [15]. Furthermore, transcriptomic SSRs may provide important information about evolution and conservational genetic variation of plant species $[8,11,16]$. Recently, different reports based on genomics [17], transcriptomics [18], metabolomics [18], and phenomics [19] have been reported in S. glabra. Various researches for characterization of extrachromosomal (chloroplast) genome [20-22] and to find the evolutionary relationship of various S. glabra subspecies [23] have also been presented. Furthermore, the amplified fragmentlength polymorphism (AFLP) and microsatellite establishment [24, 25] and SNP marker manipulation studies [26] have also been carried out for molecular evaluation in S. glabra. Nonetheless, the identification and characterization of molecular markers are still lacking in S. glabra which greatly impair establishment of breeding programs.

In this study, we generated transcriptome sequences of $S$. glabra (Thunb.) Nakai and its subspecies S. glabra ssp. brachystachys. We mined the transcriptome data to identify and characterize the SSR, SNP, and InDel markers in order to enhance the genetic resources of this pharmacologically important plant. We also reported the differentially expressed genes and their functional annotations in both subspecies.

\section{Results}

2.1. Transcriptome Sequencing and De Novo Assembly. Two S. glabra species with contrasting morphological and physiological traits (Table 1) were selected for de novo transcriptome assembly. Using the Illumina hiseq xten platform, we generated 52.327 and 57.756 million raw reads for $S$. glabra (Thunb.) Nakai (SG) and the subspecies S. glabra ssp. brachystachys (CSH), respectively (Table 2). After cleaning the reads, 7,847.65 and 8,661.75 million bases with $46.03 \%$ and $45.77 \%$ GC contents (Additional Figure 1) and 92.72\% and $92.64 \% Q>30$ were retained for $\mathrm{CSH}$ and $\mathrm{SG}$, respectively (Table 2 ).

The clean reads assembly provided 141,954 unigenes with an average length of $646.63 \mathrm{bp}$ (Table 3 ). The majority unigenes $(77,907)$ had short size $(200-400 \mathrm{bp})$ while few uni- genes (362) displayed very long size (>5,000 bp) (Figure 1(a)). The evaluation of expression quality (ExN50) of assembled contigs (N50) revealed that the majority of the contigs $(>2,000 \mathrm{bp})$ showed the high expression quantity (86-92\%) (Figure 1(b) and Additional Table 1).

The benchmarking universal single-copy orthologs (BUSCOs) were investigated, and 56.6\%, 11.2\%, and $15.4 \%$ of complete and single-copy, complete and duplicate-copy, and fragmented BUSCOs were observed, respectively (Table 3).

2.2. Transcript Annotation. The unigenes of SG and CSH after assembly were annotated using seven databases. Globally, 58,436 (41.17\%) unigenes were annotated to at least one database. We successfully assigned and annotated GO terms to $28,948(20.39 \%), 35,606$ (25.08\%), and 56,297 (39.66\%) unigenes using the Pfam, UniProt, and NR databases, respectively (Table 3). Particularly, 34,857 unigenes $(24.56 \%)$ revealed significant hits to the GO database. The GO terms classified into 147 groups and clustered in three classes and sixty subclasses (Additional Figure 2). Furthermore, $10.27 \%(14,575)$ unigenes were annotated to COG database (Additional Figure 3).

KEGG pathways analysis showed that 21,192 (14.93\%) unigenes governed the 5 main and 33 subcategories of KEGG database (Additional Figure 4). The unigenes in "carbohydrate metabolism" were further evaluated for best functional orthologous group. In total, 187 unigenes were found for glutathione S-transferase (GST) as the top functional ortholog.

The species comparison analysis revealed $41.38 \%$ similarity with 10 species. Among the similarity hits, majority (37.18\%) of the species showed $80-95 \%$ similarity. S. glabra had the highest transcript similarity with Cinnamomum micranthum f. kanehirae (9.71\%) and Nelumbo nucifera (lotus root) (8.84\%) (Additional Figure 5). The analysis of similarity distribution indicated the $37.18 \%$ sequences had $40-60 \%$ similarity, and $19.37 \%$ sequences had $80-95 \%$ similarity, while the $10.82 \%$ of sequences had $95-100 \%$ similarity (Additional Figure 6).

2.3. Expression and Pathway Enrichment Analyses. The nonstandard normal distribution was observed by transcript expression level based on FPKM values and densitydistribution pattern for each sample (Additional Figure 7A). Transcripts with similar expression levels were clustered together (Additional Figure 7C \& 7D). Pearson correlation analysis of the samples showed two separate groups for each subspecies (Additional Figure 7D), strong genetic similarity among biological replicates $\left(r^{2} \geq 0.9\right)$, while high dissimilarities were observed between CHS and SG. Among the mapped-reads, 14,584 unigenes were differentially expressed by more than twofold between GS and CHS (Additional Figure 7B). The DEGs were enriched in "chloroplast envelope," "calmodulin binding," and "carbohydrate binding" biological pathways (Figure 2). Furthermore, KEGG pathway analysis of the DEGs revealed "plant-pathogen-interaction," "endocytosis," and "plant- 
TABLE 1: Comparison of key characteristics of Sarcandra glabra (Thunb.) Nakai and S. glabra subsp. brachystachys (Blume) Verdcourt.

\begin{tabular}{lcc}
\hline Species & S. glabra & S. glabra subsp. brachystachys \\
\hline $\begin{array}{l}\text { Leaf } \\
\text { Stamen }\end{array}$ & Leathery, margin sharply coarsely serrate except basally & Papery, margin dully serrate except basally \\
Stigma & Baculate to terete, thecae shorter than connective & Ovoid, thecae almost as long as the connective \\
Fruit & Subcapitate or minutely spotted & Minutely spotted \\
\hline
\end{tabular}

TABLE 2: The summary for Sarcandra glabra de novo transcriptome assembly.

\begin{tabular}{|c|c|c|c|c|c|c|}
\hline \multirow{2}{*}{ Sample name } & \multicolumn{3}{|c|}{${ }^{*} \mathrm{CSH}$} & \multicolumn{3}{|c|}{ *SG } \\
\hline & 1 & 2 & 3 & 1 & 2 & 3 \\
\hline \multicolumn{7}{|l|}{ Raw reads } \\
\hline Total raw reads & $52,327,874$ & $50,982,388$ & $49,476,364$ & $48,520,562$ & $57,756,352$ & $55,998,238$ \\
\hline Total bases & $7,849,181,100$ & $7,647,358,200$ & $7,421,454,600$ & $7,278,084,300$ & $8,663,452,800$ & $8,399,735,700$ \\
\hline GC content & $46.03 \%$ & $46.41 \%$ & $45.76 \%$ & $45.38 \%$ & $45.76 \%$ & $46.15 \%$ \\
\hline Q20 & $97.62 \%$ & $97.63 \%$ & $97.60 \%$ & $97.63 \%$ & $97.61 \%$ & $97.56 \%$ \\
\hline Q30 & $92.72 \%$ & $92.74 \%$ & $92.66 \%$ & $92.69 \%$ & $92.64 \%$ & $92.54 \%$ \\
\hline \multicolumn{7}{|l|}{ Clean read } \\
\hline Total reads & $52,317,712$ & $50,972,536$ & $49,465,478$ & $48,512,702$ & $57,745,284$ & $55,984,978$ \\
\hline Total bases & $7,847,656,800$ & $7,645,880,400$ & $7,419,821,700$ & $7,276,905,300$ & $8,661,792,600$ & $8,397,746,700$ \\
\hline GC content & $46.03 \%$ & $46.41 \%$ & $45.76 \%$ & $45.38 \%$ & $45.77 \%$ & $46.15 \%$ \\
\hline Q20 & $97.63 \%$ & $97.63 \%$ & $97.60 \%$ & $97.64 \%$ & $97.61 \%$ & $97.56 \%$ \\
\hline Q30 & $92.72 \%$ & $92.74 \%$ & $92.67 \%$ & $92.70 \%$ & $92.64 \%$ & $92.54 \%$ \\
\hline
\end{tabular}

${ }^{*}$ CSH stands for Sarcandra glabra ssp. brachystachys, while SG represents Sarcandra glabra (Thunb.) Nakai.

hormone signal-transduction" as the top enriched pathways (Figure 2).

2.4. Identification and Characterization of SSRs in the Transcriptomes. Only the unigenes with FPKM $\geq 1$ were employed for detecting and SSRs. Collectively, 52,317,712 clean reads were assembled to 141,954 contigs of an average contig length of $646.63 \mathrm{bp}$. A total of 35,423 microsatellites were recognized on 25,620 contigs (Table 4 and Additional Table 2). The SSR density in the transcriptome was 385.91 SSRs/Mb. Among the SSRs, mononucleotide microsatellites were the most abundant $(47.77 \%)$ with $16,920 \mathrm{bp}$ length, followed by di- $(33.85 \%)$, tri- $(16.59 \%)$, tetra- (1.1\%), hexa$(0.45 \%)$, and pentanucleotide $(0.25 \%)$ types. The mononucleotide motifs A (50\%) and T (48\%) were highly abundant repeats. Among the total, 1,968 dinucleotide motifs GA (17.63\%) and AG (16.61\%) were the most detected types, while only 10 GC and 5 CG motifs were available in the transcriptome data. Among the trinucleotide, ATG (6.77\%) followed by TCT (6.25\%), and GAA $(6.03 \%)$ motif types were identified, while only 8 CCG and 9 GCG were observed.

An average microsatellite length of S. glabra was $23.7 \mathrm{bp}$. The repeat motif size significantly affects the length variation of microsatellites. Hexanucleotide motifs with an average length of $34.8 \mathrm{bp}$ were the longest motif followed by penta(27.72 bp), di- (24.27 bp), tetra- (21.92 bp), and trinucleotide (19.7 bp) motifs. The mononucleotides showed the shortest average motif length of $12.56 \mathrm{bp}$. A trinucleotide motif 87 bp length and 29-fold repetition was the longest microsatel- lite observed (Table 4 and Additional Table 2). Other than these motifs, a compound motif type denoted as "c" for which two types of motifs separated by a few nonrepeating nucleotides, and " $\mathrm{c}$ " " motifs with two different types of repeats without any separation were observed also. The 9.08\% (3218) and $0.25 \%$ (90) of total SSRs were " $c$ " and " $c$ " type compound motifs, respectively, and showed an average length of $76.67 \mathrm{bp}$ and $47.1 \mathrm{bp}$. Among the compound motifs, the longest was of 481 bp with 4 mononucleotide and 4 hexanucleotide motifs with multiple repeats. In the top ortholog group of carbohydrate metabolic pathway (K00799), a total 14 SSRs were identified for 19 assembled unigenes.

The widest range was observed for mononucleotides (10$80)$ in repetitions, while this range for di-, tri-, tetra-, penta-, and hexanucleotide microsatellites was 6 to 42,5 to 29,5 to 16,5 to 8 , and 3 to 11 , respectively. Most of the motifs $(5,749)$ had 10 repeats followed by 11 repeat motifs $(3,854)$. The majority of unigenes $(81 \%, 20,756)$ possessed only one microsatellite motif, while $15 \%$ (4,036), 2.68\% (687), $0.46 \%$ (119), 17, and 4 unigenes had 2, 3, 4, 5, and 6 SSR motifs. Only one of the unigenes observed had nine SSR motifs.

2.5. SNPs and InDel Variant Identification, Distribution, and Their Selection Signature. We detected in the unigenes a total of 726,476 single nucleotide polymorphism sites (SNP) with an average of one SNP in $92.32 \mathrm{bp}$. These SNP markers were situated in 65,539 unigenes. The total number of transition and transversion mutations was 1,588,169 (63\%) and 928967 (37\%) (Table 5 and Additional Table 3). A total of 
TABLE 3: Characteristic descriptive and the functional annotation of de novo transcriptome assembly of Sarcandra glabra.

\begin{tabular}{lc}
\hline Descriptive & Value \\
\hline Total length (bp) & $91,791,960$ \\
Total unigene & 141,954 \\
GC contents (\%) & 41.91 \\
N50 (bp) & 989 \\
N90 (bp) & 264 \\
Average (bp) & 646.63 \\
Median (bp) & 363 \\
Minimum (bp) & 201 \\
Maximum (bp) & 17087 \\
Contigs of size $<600$ bp & 77907 \\
Contigs of size $\geq 600$ bp & 22669 \\
Contigs of size $\geq 1000$ bp & 15536 \\
Contigs of size $\geq 2,000$ bp & 362 \\
${ }^{*}$ Complete BUSCOs & $291(67.8 \%)$ \\
Complete and single-copy BUSCOs & $243(56.6 \%)$ \\
Complete and duplicated BUSCOs & $48(11.2 \%)$ \\
Fragmented BUSCOs & $66(15.4 \%)$ \\
Missing BUSCOs & $72(16.8 \%)$ \\
Total BUSCO groups searched & $429(100 \%)$ \\
Total annotations & $58,436(41.17 \%)$ \\
1: UniProt & $35,606(25.08 \%)$ \\
2: Pfam & $28,948(20.39 \%)$ \\
3: GO & $34,857(24.56 \%)$ \\
4: KEGG & $21,192(14.93 \%)$ \\
5: COG pathway & $14,575(10.27 \%)$ \\
7: EggNOG & $23,086(16.26 \%)$ \\
Complete BUSCOs, & $56,297(39.66 \%)$ \\
\hline
\end{tabular}

${ }^{*}$ Complete BUSCOs: the detected gene length within the $95 \%$ confidence interval of the average length in the BUSCO homologous group, it may with single or multiple copies, while the incomplete BUSCOs are denoted as fragmented, and undetected BUSCO homologous group is denoted as missing.

42,939 insertion and deletion (InDels) were recorded in the $S$. glabra assembled transcriptome. Out of them, 18,607 (43\%) were insertions while 24,332 (57\%) were deletion mutations. On an average, one InDel per $746 \mathrm{bp}$ was found in 19,153 unigenes (Table 5 and Additional Table 4). In total, 89 SNP markers for 89 unigenes were related to 9 GO terms including sulfur compound metabolic process, response to stress, signal transduction, molecular functions, oxidoreductase-activity, transferase-activity transferringalkyl or -aryl (other than methyl) groups, cellular components, cytoplasm, and nucleus, in the top ortholog group (K00799) of carbohydrate metabolic pathway (Additional Table 5).

The genetic variant (SNPs and Indels) distribution and the effect on gene functions were investigated. Totally, $36.894 \%(275,669)$ variants were observed in exon regions while $20.726 \%(154,861)$ variants were in intergenic regions. The variant numbers in $5^{\prime}$ UTR and $3^{\prime}$ UTR were $19.327 \%$ $(144,409)$ and $23.051 \%(172,232)$, respectively. Only 22 vari- ants were found in the splice site regions. The majority of variants $(60.33 \%)$ have modifying effect in genome, which consisted of $3^{\prime} \mathrm{UTR}, 5^{\prime} \mathrm{UTR}$, and intergenic variants. In contrast, $19.934 \%$ and $19 \%$ variants could exert low and moderate effects, respectively (Table 5). The missense variants were observed to be moderate effect variants. The start- and stopcodon-loss-in-function and stop-codon-gain-in-function variants were observed to have the high effect in the genome. The variants within the coding area mostly missense $(52.116 \%)$ or silent $(46.482 \%)$ while only $1.42 \%$ mutations were nonsense variations.

2.6. Validation of SSR Markers. Most of the two ends of SSRs are conservative single-copy sequences. The primers were designed as per complementary sequences of the two ends of the SSRs. A total of sixteen SSR markers including two markers for each type of SSRs were randomly selected for validation. Fifteen SSR markers were successfully amplified in 26 to 30 tested genotypes, while one marker was validated in 19 genotypes. The length polymorphism of SSR sites was displayed by electrophoresis of PCR products. One to 17 alleles could be identified. The description of validated markers, their PCR products, and polymorphic alleles are presented in Additional Table 6.

\section{Discussion}

The genetic improvement of S. glabra as a pharmacologically important plant is required, which could be accelerated by breeding strategies employing molecular markers. Two SG species (S. glabra ssp. brachystachys and S. glabra (Thunb.) Nakai) have various morphological differences including plant height, leaf shape, and appearance of inflorescences between them [27], and both are considered as model in previous studies for this family [28]. A molecular plant breeding program is always a fast and cost-effective tool in genomic improvement but it demands a rich molecular marker resource that is deficient for S. glabra. Development of such markers is not easy as its genome has not been sequenced yet. The transcriptome-based markers characterized in the study can be used to identify the linked and/or cosegregating loci for linkage and association-based genome mapping of various traits of interest in S. glabra as reported in various other plant species [29].

High-throughput sequencing of transcriptome is a fast and cost-effective way to characterize the genes and identify polymorphic molecular markers in nonmodel plants [12]. In the current study, we obtained the transcriptome data of S. glabra and revealed the available unigenes by de novo assembly. The high N50 and ExN90 values revealed the excellent sequencing quality. The coverage of functional genes was expressed by the BUSCO percentage. The relatively low percentage of complete BUSCOs may indicate the possibility that all genes in this species were not captured. It is normal as only the leaf samples and a single genotype per species were used in this study. It is known that the gene content and expression are different across tissues and genotypes. Furthermore, the transcriptome sequencing reveals only expressed functional genes, while a lot of genes present 


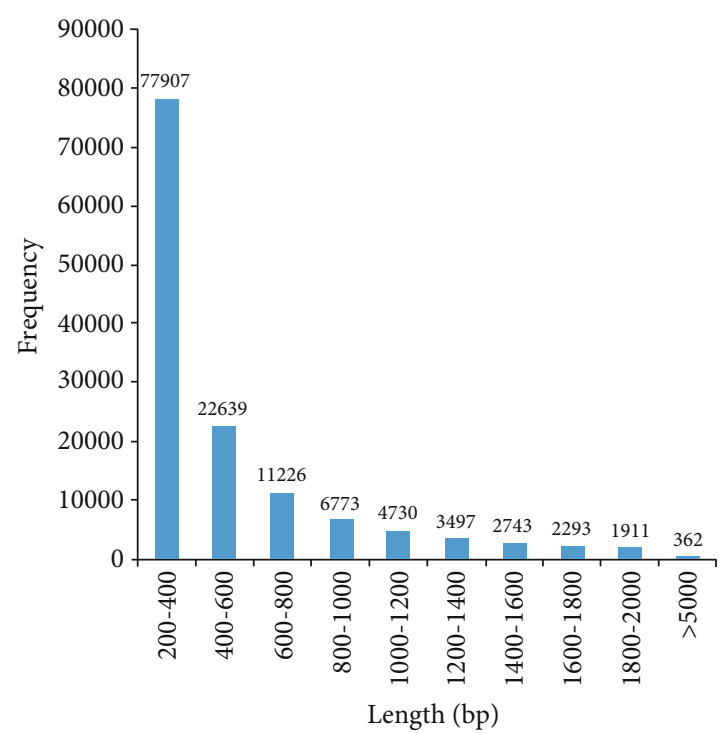

(a)

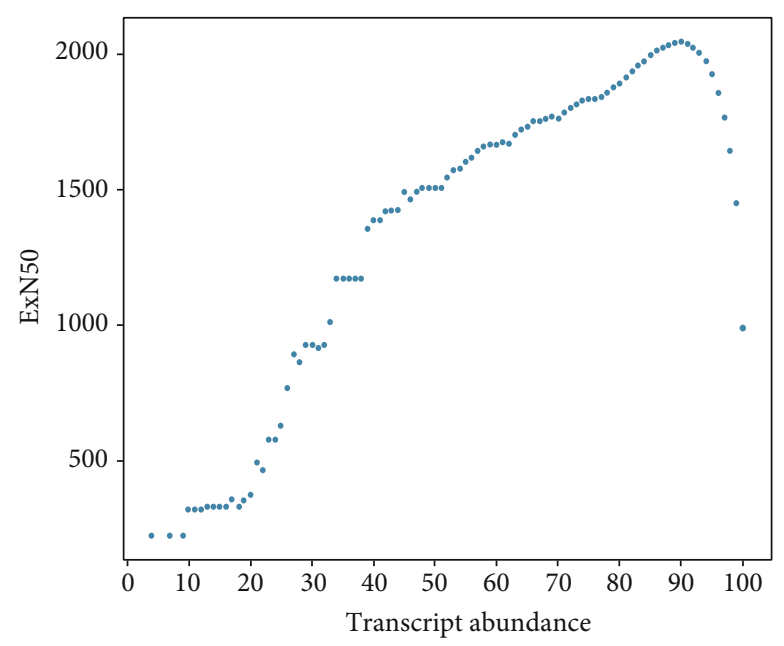

(b)

FIGURE 1: The clean reads after the sequence assembly: (a) length-based distribution of assembled reads and (b) distribution of N50 on the bases of expression clustering from $0-100$.

in the genome are missing. On the basis of transcriptome assembly, we characterized the molecular (microsatellite, SNP, and InDel) markers. The results indicated the availability of microsatellites in about one-fifth of the transcripts (18.04\%), which is quite higher than the estimation of $2-5 \%$ in other reported studies [30]. Besides SSRs, we also detected SNPs and Indels which are considered very valuable markers. Recently, the development, characterization, and utilization of these markers in model crops as rice [31] and nonmodel crops as guar potato $[11,32]$ have been reported. The density of InDel and SNP identification was higher than the other studies as 17.10 SNPs per Kb in Cyamopsis tetragonoloba $\mathrm{L}$. Taub [2] and one SNP per $17.08 \mathrm{~Kb}$ in Guar [32]. Collectively, the molecular markers obtained in this study will be helpful for future genomics studies and markers assisted selection in S. glabra. The leaf samples were employed for transcriptome characterization in this study because leaves are the main source of bioactive ingredients in SG. Nonetheless, mixing different tissues for transcriptome analysis may be a better approach in order to identify more transcripts and potentially more molecular markers as described in previous studies [33,34].

The identified molecular markers in this study have tagged functional genes governing important metabolic activities including the ion binding. Various reports have shown the roles of ion channels in cancer from two principal standpoints: examining how specific ion channels are involved in certain cancer-related cellular behaviors such as proliferation, apoptosis, migration, or angiogenesis or examining the specific expression and functional profiles of various channel characteristic of certain human cancers [35]. Among the annotated pathways, the carbohydrate metabolism was observed to be on top hit. The evaluation of functional ortholog of this pathway indicated 187 unigenes for the glutathione S-transferase (GST) functional ortholog (k00799 in Map-05200) known as important genes in medic- inal plants [22]. The glutathione S-transferases belong to a supergene family of seven cytosolic enzymes that catalyze the conjugation of glutathione (GSH) to a variety of electrophiles including arene oxides, unsaturated carbonyls, organic halides, and other substrates [35]. In humans, polymorphism in GST genes has been associated with susceptibility to various diseases. Previous studies described the associations between GST mutants (GSTM1 null and GSTT1 null) and location of colorectal cancers in individuals with mutation 1 in the MLH1 mismatch repair gene in Finnish kindreds. It is proposed that the GST genes are among other detoxicating enzymes that act as modifying genes and affect the phenotype in monogenic colorectal cancer [35].

Even though the establishment of AFLP and microsatellite markers have been reported in recent studies [24], and important molecular marker resources of $S$. glabra have been generated in this study, it is still less sufficient as compared to the well-studied model plants. Further works based on longread transcriptome sequencing as well as whole genome sequences are needed to accelerate the genetic improvement of this important crop.

\section{Methods}

4.1. Plant Materials, Growth Conditions, and RNA Extraction. The seeds of Sarcandra glabra (Thunb.) Nakai (SG) and its subspecies S. glabra ssp. brachystachys (Figure 3) were obtained from Hainan Branch Institute of Medicinal Plant Development, Chinese Academy of Medical Sciences, China. The selected, cleaned, and healthy seeds were grown in pots in a greenhouse with temperature 22$30^{\circ} \mathrm{C}$ and relative humidity $55-65 \%$. The 30 -day-old welldeveloped single plants were selected for RNA-seq study. Leaf tissues of SG and CSH were collected from three selected individual plants. The fresh tissues $(100 \mathrm{mg})$ were used to extract the total RNA. The extracted RNA was further treated 


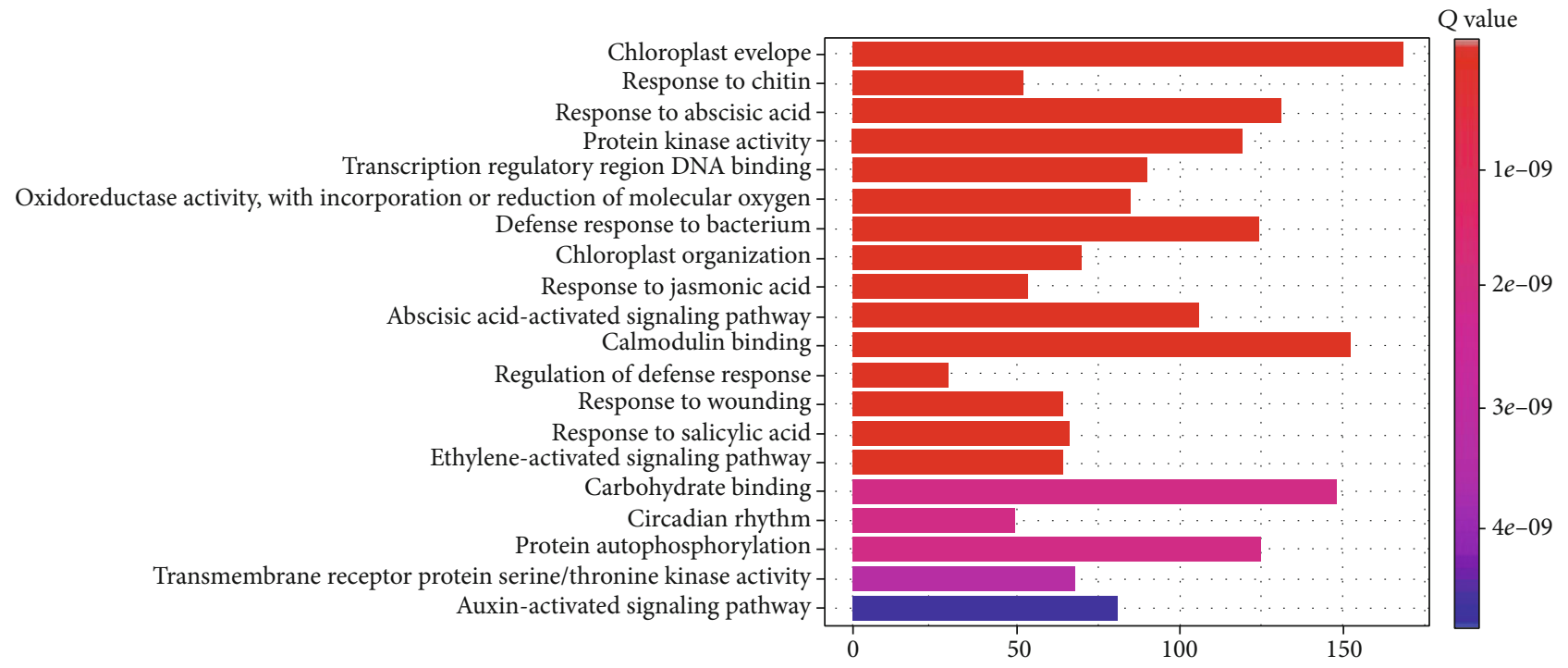

(a)

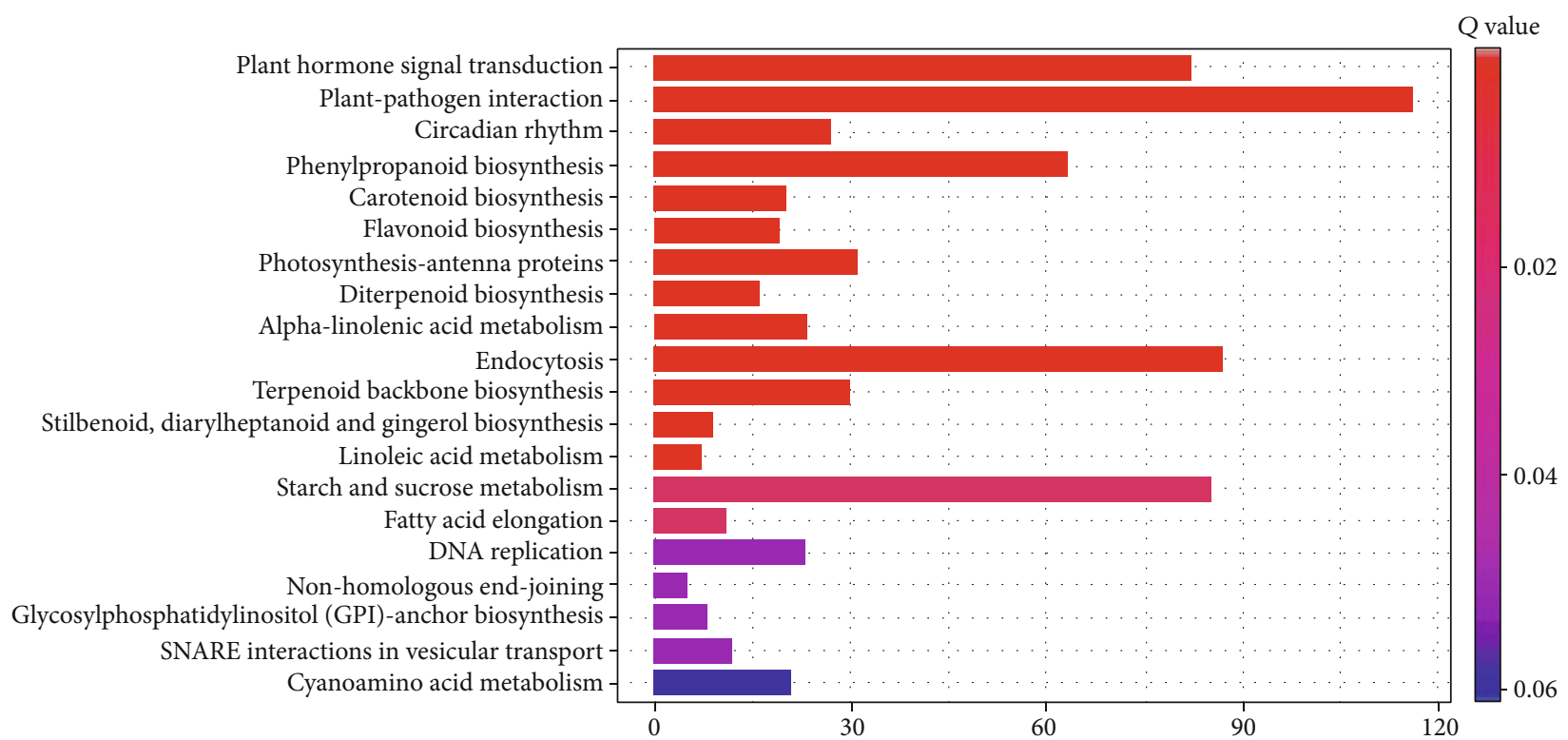

(b)

FIGURE 2: Classification of differentially expressed gene for functional annotations: (a) on the basis of Gene Ontology database (GO) and (b) through pathway significant enrichment database Kyoto Encyclopedia of Genes and Genomes (KEGG).

with DNase I for purification. The step by step process as total RNA sample detection, mRNA enrichment, doublestrand cDNA-synthesis, end repair, splice selection and PCR-based amplification library quality detection, and computer sequencing was performed at Wuhan Benagen Tech Solutions Company Limited, Wuhan, China, to generate the paired-end reads.

4.2. Transcriptome Sequencing, Cleaning, and Assembly. The preparation of cDNA library and sequencing was performed by using three independent biological replicates for SG and CSH. The total RNA from each sample was extracted, and cDNA library was prepared. The original files of image data were obtained by high-throughput sequencing (Illumina hiseq xten) and were transformed into pair-end raw reads by base-calling analysis. As per machine's sequencing strategy, $150 \mathrm{bp}$ average read length was maintained. The raw reads with joint sequences and/or $<5$ mass value, $\geq 50 \%$ proportion rate, more than or equal to $5 \% \mathrm{~N}$ base (the base with undetermined information), containing Poly-A, were filtered out to get the cleaned reads. The sequencing quality was estimated by $Q$ Phred values. The clean reads were assembled into a fastq format [36]. The Trinity v 2.6.6 program [37] was used for transcriptome assembly and to get the unigenes with default parameters. The accuracy and effectiveness of the assembly results were ensured by estimation of N50, ExN50, and BUSCO [38].

4.3. Clustering and Functional Annotation. The extracted unigenes were manipulated by the Transcoder software $\mathrm{v}$ 
TABLE 4: SSR motif repeat distribution in transcriptome data of Sarcandra glabra.

\begin{tabular}{|c|c|c|c|c|c|c|c|c|c|}
\hline \multirow{2}{*}{ Number of repeats } & \multicolumn{6}{|c|}{ SSR motifs } & \multicolumn{2}{|c|}{$\begin{array}{l}\text { Compound } \\
\text { motifs }\end{array}$} & \multirow[t]{2}{*}{ Total } \\
\hline & Mono & Di & Tri & Tetra & Penta & Hexa & c & $c^{*}$ & \\
\hline 5 & 0 & 0 & 2113 & 224 & 48 & 65 & & & 2450 \\
\hline 6 & 0 & 1721 & 1004 & 62 & 8 & 41 & & & 2836 \\
\hline 7 & 0 & 1085 & 559 & 6 & 7 & 15 & & & 1672 \\
\hline 8 & 0 & 897 & 363 & 7 & 5 & 6 & & & 1278 \\
\hline 9 & 0 & 665 & 99 & 1 & 0 & 1 & & & 766 \\
\hline 10 & 5133 & 505 & 104 & 6 & 0 & 1 & & & 5,749 \\
\hline 11 & 2717 & 1043 & 93 & 0 & 0 & 1 & & & 3,854 \\
\hline 12 & 1808 & 267 & 67 & 0 & 0 & 0 & & & 2142 \\
\hline 13 & 1096 & 116 & 63 & 1 & 0 & 0 & & & 1276 \\
\hline 14 & 880 & 141 & 45 & 0 & 0 & 0 & & & 1066 \\
\hline 15 & 687 & 138 & 31 & 0 & 0 & 0 & & & 856 \\
\hline 16 & 541 & 142 & 35 & 1 & 0 & 0 & & & 719 \\
\hline 17 & 390 & 138 & 11 & 0 & 0 & 0 & & & 539 \\
\hline 18 & 266 & 170 & 4 & 0 & 0 & 0 & & & 440 \\
\hline 19 & 217 & 157 & 6 & 0 & 0 & 0 & & & 380 \\
\hline 20 & 173 & 170 & 2 & 0 & 0 & 0 & & & 345 \\
\hline$>20$ & 486 & 1,312 & 9 & 0 & 0 & 0 & & & 1807 \\
\hline Total & 14394 & 8667 & 4608 & 308 & 68 & 130 & 3218 & 90 & 31483 \\
\hline
\end{tabular}

TABLE 5: Distribution of SNP and insertion and deletion variants in the Sarcandra glabra genome.

(a)

\begin{tabular}{|c|c|c|c|c|c|}
\hline \multirow{2}{*}{$\begin{array}{l}\text { Type of SNP variants } \\
\text { Type }\end{array}$} & \multirow[b]{2}{*}{ Count } & \multirow[b]{2}{*}{ Ratio } & \multicolumn{3}{|c|}{ Type of InDel variants } \\
\hline & & & Type & Count & Ratio \\
\hline 3'UTR variant & 172,232 & $23.05 \%$ & $3^{\prime} \mathrm{UTR}$ variant & 14,938 & $32.21 \%$ \\
\hline $5^{\prime} \mathrm{UTR}$ premature start codon gain variant & 20,696 & $2.77 \%$ & $5^{\prime} \mathrm{UTR}$ variant & 12,609 & $27.19 \%$ \\
\hline $5^{\prime}$ UTR variant & 123,713 & $16.56 \%$ & Conservative in-frame deletion & 1,195 & $2.58 \%$ \\
\hline Initiator codon variant & 94 & $0.01 \%$ & Conservative in-frame insertion & 789 & $1.70 \%$ \\
\hline Intergenic region & 154,861 & $20.73 \%$ & Disruptive in-frame deletion & 1,312 & $2.83 \%$ \\
\hline Missense variant & 142,027 & $19.01 \%$ & Disruptive in-frame insertion & 871 & $1.88 \%$ \\
\hline Splice region variant & 25 & $0.00 \%$ & Frame-shift variant & 6,919 & $14.92 \%$ \\
\hline Start lost & 520 & $0.07 \%$ & Intergenic region & 5,782 & $12.47 \%$ \\
\hline Stop gained & 3,865 & $0.52 \%$ & Splice region variant & 2 & $0.00 \%$ \\
\hline Stop lost & 1,027 & $0.14 \%$ & Start lost & 471 & $1.02 \%$ \\
\hline Stop retained variant & 318 & $0.04 \%$ & Stop gained & 237 & $0.51 \%$ \\
\hline Synonymous variant & 127,819 & $17.11 \%$ & Stop lost & 1,257 & $2.71 \%$ \\
\hline
\end{tabular}

(b)

\begin{tabular}{|c|c|c|c|c|c|}
\hline \multicolumn{3}{|l|}{ Region wise } & \multicolumn{3}{|c|}{ Region wise } \\
\hline Exon & 275,669 & $36.89 \%$ & Exon & 11,086 & $24.96 \%$ \\
\hline Intergenic & 154,861 & $20.73 \%$ & Intergenic & 5,782 & $13.02 \%$ \\
\hline Splice sites & 22 & $0.00 \%$ & Splice sites & 1 & $0.00 \%$ \\
\hline $3^{\prime} \mathrm{UTR}$ & 172,232 & $23.05 \%$ & $3^{\prime} \mathrm{UTR}$ & 14,938 & $33.63 \%$ \\
\hline $5^{\prime} \mathrm{UTR}$ & 144,409 & $19.33 \%$ & $5^{\prime} \mathrm{UTR}$ & 12,609 & $28.39 \%$ \\
\hline
\end{tabular}




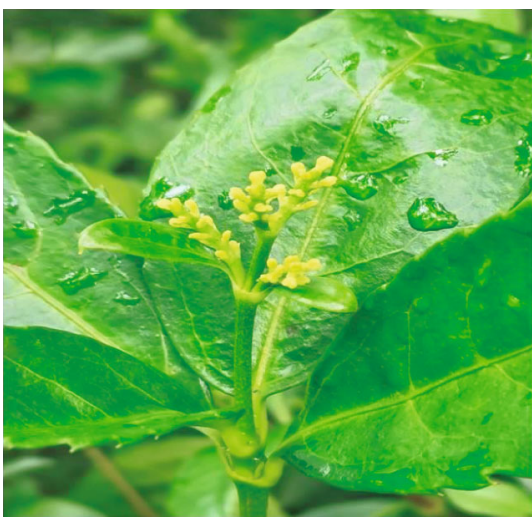

(a)

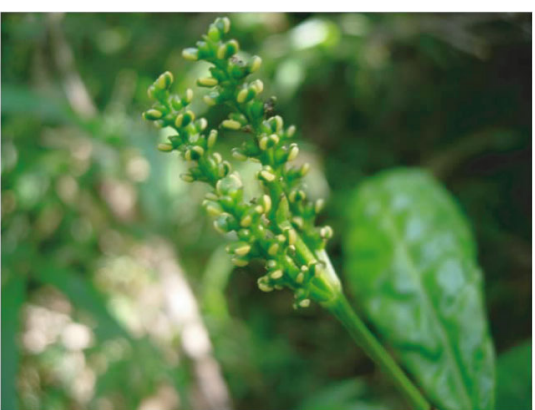

(c)

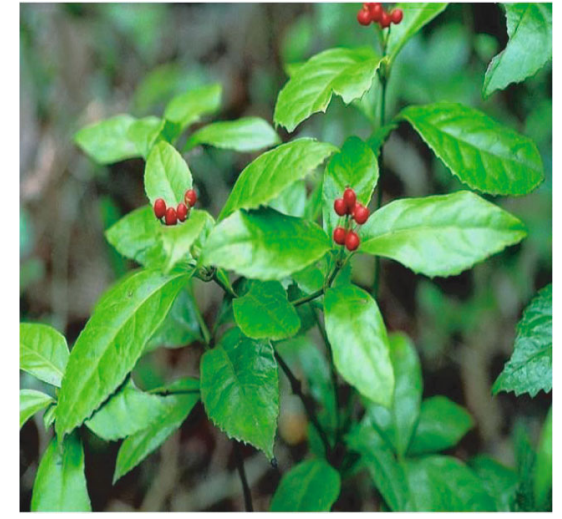

(b)

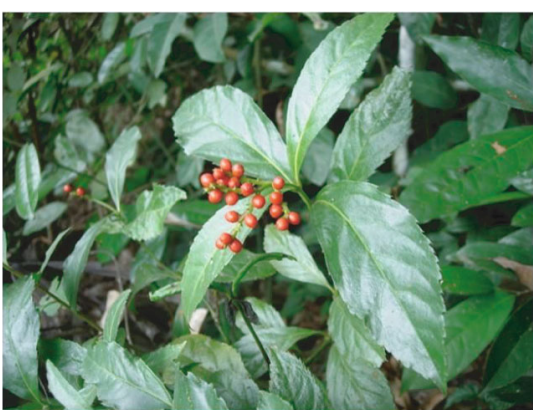

(d)

Figure 3: Morphological appearance of ( $a, c)$ inflorescence and (b, d) leaves of (a, b) Sarcandra glabra (Thunb.) Nakai and (c, d) subspecies S. glabra ssp. brachystachys.

4.1.0 to predict and translate the reading frames with default parameters. The blast X and blast $\mathrm{P}$ tools of diamond $\mathrm{v} 0.9 .24$ program [39] were used to compare the unigene sequences and protein sequences against UniProt database [40] with default parameters. At the same time, diamond v 0.8.36 program blast $\mathrm{X}$ was used to compare the unigene sequence with NR database to find the closely related species. Functional annotation was further performed by mapping unigenes to UniProt/Swiss port, Kyoto Encyclopedia of Genes and Genomes (KEGG) [41], EggNog [42], and Gene Ontology (GO) $[43,44]$ databases. The Hmmscan v3.1 (parameter: $E$ value 0.01 ) was used for protein domain prediction on the basis of Pfam values.

4.4. Mining and Validation Analysis of SSRs. The MIcroSAtellite identification tool (MISA) v 1.0 was used to detect SSR loci from assembled contigs (http://pgrc.ipk-gatersleben.de/ $\mathrm{misa} / \mathrm{misa}$.html). The minimum/standard repetition parameter was 10 for mono-, 6 for di-, and 5 for tri-, tetra, penta-, and hexanucleotide microsatellite motifs, respectively. The compound microsatellites were defined if the distance between two repeated motifs was shorter than 100 nucleotides [32]. Three primer pairs for all SSR markers were designed by primer 3 v2.3.5 [45] with default parameters. After SSR marker identification, sixteen SSR markers were randomly selected for further validation and a fixed sequence (GTAAAACGACGGCCAGT) was designed at the 5 ends of each of primer to match the fluorescent-labeled primer. PCR amplification with the designed primers of selected SSR markers was performed for DNA from fresh leaves of 31 genotypes including CSH4, CSH5, CSH6, CSH7, CSH8, SG31, SG32, SG33, SG34, SG42, SG44, SG45, SG48, SG49, SG50, SG53, SG55, SG2, SG4, SG5, SG13, SG14, SG15, SG16, SG18, SG19, SG20, SG23, SG28, and SG29 using DNeasy Plant Mini Kit (QIAGEN). All samples were obtained from College of Pharmacy, Jiangxi University of Traditional Chinese Medicine, China. After amplification, the fragment size was analyzed by Applied Biosystems 3737 sequencer.

4.5. Identification of SNPs and InDels. To identify the putative single nucleotide polymorphic sites (SNPs) and insertion and deletion events (InDels) in the transcripts, the highquality transcriptome assembled sequences were aligned and compared by STAR v2.7.0 d program [46] with default parameters, and the BAM format comparison files were obtained. The Picard tool (version: 1.93) software package was employed to compare and process the results with default parameters. The SNP calling and low-quality filtering were performed by "haplotypecaller" in GATK (version: 4.1.4) [47] using default parameters. The SNP annotation was performed by "snpeff (version 4.3)" [48] using default parameters. Furthermore, the manual characterization of obtained SNP and InDel markers was performed.

4.6. Expression Analysis and Mining the Differentially Expressed Genes. The bowtie2 v 2.3.4 [49] with zero mismatch parameter was used to compare the reads to the 
assembled transcriptome. The results were compared with RSEM 2 (v 1.3.1) [50]. The number of read count on each gene was obtained from each sample, and the gene expression level was estimated by the FPKM method. The difference gene expression was analyzed by comparing read count data from the two subspecies using the DESeq2 program with the $Q$ value $<0.05$ and $\log 2 \mathrm{FC}>1$ as a threshold [35].

\author{
Abbreviations \\ BUSCO: Benchmarking universal single-copy ortholog \\ $\mathrm{CSH}: \quad$ S. glabra ssp. brachystachys \\ FPKM: Fragments per kilobase of transcript per million \\ mapped reads \\ GBS: Genotyping-by-sequencing \\ InDels: Insertion and deletion events \\ SG: $\quad$ S. glabra (Thunb.) Nakai \\ SNP: $\quad$ Single nucleotide polymorphism \\ SSR: $\quad$ Simple sequence repeat.
}

\section{Data Availability}

The RNA-seq data has been submitted to NCBI SRA: PRJNA671629.

\section{Disclosure}

The funder has no role in the study design, data collection and analysis, decision to publish, or preparation of the manuscript.

\section{Conflicts of Interest}

The authors declare that they have no competing interests.

\section{Authors' Contributions}

Y. X. and Y. Z. conceived and designed the study. Y. X., S. T., R. L., X. H., F. L., F. G., and W. H. collected plant materials and conducted the experiment and data analysis. Y. X. wrote the manuscript. Y. Z. provided funding and revised the first drafts of the manuscript. All authors have read and approved the final version of this manuscript.

\section{Acknowledgments}

This work was supported by the National Natural Science Foundation of China (82060684 and 31700626), the Jiangxi University of Traditional Chinese Medicine 1050 Young Talents Project (5141900107), the Young and middle-aged Talents Training Program of Traditional Chinese Medicine of Jiangxi Province (2020-2), the National Science Foundation of Jiangxi Province (20181BAB205075), and the First-class Discipline of Chinese Medicine Program of Jiangxi University of Traditional Chinese Medicine (JXSYLXKZHYAO023). Special thanks are due to Dr. Zheng Xilong for his help for the collection of Sarcandra glabra ssp. brachystachys. Thanks are due to Dr. Zheng Xilong, Dr. Li Haitao, and Ms. Du Xiaolang for providing photos of Sarcandra glabra ssp. brachystachys.

\section{Supplementary Materials}

Additional Figure 1: the base distribution of sample's reads. The first half is the base content distribution of the first end of the double end sequencing sequence, and the second half is the base content distribution of the other end. Additional Figure 2: simplified annotation information of Gene Ontology classification of identified transcripts for S. glabra. Additional Figure 3: the gene classification on the bases of similarity on cluster of indigenous groups of protein database for S. glabra. Additional Figure 4: the gene classification on the bases of KEGG metabolic pathways for S. glabra. Additional Figure 5: the comparison of S. glabra sequence with the other species, and the results for top 10 species have been presented while rests are classified into other species. Additional Figure 6: the transcriptome sequence evaluation for S. glabra by (A) comparison of evaluation values ( $E$ values) and (B) the sequence consistency analysis. Additional Figure 7: expression analysis: (A) The FPKM density map shows the overall gene expression pattern of each sample; (B) the MA plot displays the differentially expressed genes; (C) the expression patterns based on cluster analysis of all the DEGs in the different samples; (D) the correlation between samples based on DEGs. Additional Table 1: expression-based frequency distribution of transcripts extracted from transcriptome data of S. glabra. Additional Table 2: characterization of transcriptome SSR markers in S. glabra. Additional Table 3: characterization of transcriptome SNP markers in S. glabra. Additional Table 4: characterization of transcriptome InDel markers in S. glabra. Additional Table 5: the SSR, SNP, and InDel markers in transcriptome-based unigenes of $S$. glabra with the respective annotations in GO, KEGG, and COG databases. Additional Table 6: list of sixteen randomly selected and validated SSR markers, their primers, and PCR products. (Supplementary Materials)

\section{References}

[1] H.-J. Cao, R.-R. Tan, R.-R. He et al., "Sarcandra glabra extract reduces the susceptibility and severity of influenza in restraintstressed mice," Evidence-Based Complementary Alternative Medicine, vol. 2012, article 236539, pp. 1-12, 2012.

[2] H. Li, Z. Wang, X. Sun, C. Pan, X. Gao, and W. Liu, "Chemical and rheological properties of proteoglycans from Sarcandra glabra (Thunb.) Nakai," International Journal of Biological Macromolecules, vol. 132, pp. 641-650, 2019.

[3] N. Pharmacopoeia, Pharmacopoeia of the People's Republic of China, People's Medical Publishing House, Beijing, China, 2005.

[4] S. Yaermaimaiti, P. Wang, J. Luo, R.-J. Li, and L.-Y. Kong, "Sesquiterpenoids from the seeds of Sarcandra glabra and the potential anti-inflammatory effects," Fitoterapia, vol. 111, pp. 7-11, 2016.

[5] X. Guo, L. Shen, Y. Tong et al., "Antitumor activity of caffeic acid 3,4-dihydroxyphenethyl ester and its pharmacokinetic and metabolic properties," Phytomedicine, vol. 20, no. 10, pp. 904-912, 2013.

[6] L. Jin, X. Guan, W. Liu et al., "Characterization and antioxidant activity of a polysaccharide extracted from Sarcandra glabra," Carbohydrate Polymers, vol. 90, no. 1, pp. 524-532, 2012. 
[7] W. Liu, W. Lu, Y. Chai, Y. Liu, W. Yao, and X. Gao, "Preliminary structural characterization and hypoglycemic effects of an acidic polysaccharide SERP1 from the residue of Sarcandra glabra," Carbohydrate Polymers, vol. 176, pp. 140-151, 2017.

[8] R. Yasodha, R. Vasudeva, S. Balakrishnan et al., "Draft genome of a high value tropical timber tree, Teak (Tectona grandis L. $\mathrm{f}$ ): insights into SSR diversity, phylogeny and conservation," DNA Research, vol. 25, no. 4, pp. 409-419, 2018.

[9] M. K. Vishwakarma, S. M. Kale, M. Sriswathi et al., "Genomewide discovery and deployment of insertions and deletions markers provided greater insights on species, genomes and sections relationships in the genus Arachis," Frontiers in Plant Science, vol. 8, 2017.

[10] S. Park, S. Son, M. Shin, N. Fujii, T. Hoshino, and S. Park, "Transcriptome-wide mining, characterization, and development of microsatellite markers in Lychnis kiusiana (Caryophyllaceae)," BMC Plant Biology, vol. 19, no. 1, p. 14, 2019.

[11] Y. Wang, M. A. R. Rashid, X. Li et al., "Collection and evaluation of genetic diversity and population structure of potato landraces and varieties in China," Frontiers in Plant Science, vol. 10, p. 139, 2019.

[12] J. E. Zalapa, H. Cuevas, H. Zhu et al., "Using next-generation sequencing approaches to isolate simple sequence repeat (SSR) loci in the plant sciences," American Journal of Botany, vol. 99, no. 2, pp. 193-208, 2012.

[13] R. K. Varshney, A. Graner, and M. E. Sorrells, "Genic microsatellite markers in plants: features and applications," Trends in Biotechnology, vol. 23, no. 1, pp. 48-55, 2005.

[14] Y. C. Li, A. B. Korol, T. Fahima, A. Beiles, and E. Nevo, "Microsatellites: genomic distribution, putative functions and mutational mechanisms: a review," Molecular Ecology, vol. 11, no. 12, pp. 2453-2465, 2002.

[15] G. M. Cordeiro, R. Casu, C. L. McIntyre, J. M. Manners, and R. J. Henry, "Microsatellite markers from sugarcane (Saccharum spp.) ESTs cross transferable to erianthus and sorghum," Plant Science, vol. 160, no. 6, pp. 1115-1123, 2001.

[16] Y. Kashi and D. G. King, "Simple sequence repeats as advantageous mutators in evolution," Trends in Genetics, vol. 22, no. 5, pp. 253-259, 2006.

[17] J. Chen, D. Tohty, and W. Guo, "A comparative study of five methods of the genomic DNA isolation from Sarcandra glabra (Thunb.) Nakai leaves," Journal of Xinjiang Normal University (Natural Sciences Edition), vol. 1, 2008.

[18] D. Xie, L. Chen, C. Zhou et al., "Transcriptomic and metabolomic profiling reveals the effect of LED light quality on morphological traits, and phenylpropanoid-derived compounds accumulation in Sarcandra glabra seedlings," BMC Plant Biology, vol. 20, no. 1, p. 476, 2020.

[19] C. Zhou, D. Guo, and F. Ni, "Comparative study on ultrasonic extraction and reflux extraction of rosmarinic acid in stem of Sarcandra glabra (Thunb.) Nakai," Journal of Fujian University of Traditional Chinese Medicine, vol. 1, 2012.

[20] W. Wang, P. Zou, G. Liu, and S. Dai, "Characterization of the complete chloroplast genome sequence of Sarcandra glabra (Chloranthales)," Mitochondrial DNA Part B, vol. 5, no. 1, pp. 864-865, 2020.

[21] E.-K. Han, W.-B. Cho, G. Choi, and J.-H. Lee, "The complete chloroplast genome of Sarcandra glabra (Chloranthaceae): a perianthless basal angiosperm," Mitochondrial DNA Part B, vol. 3, no. 2, pp. 661-662, 2018.
[22] X. Liu, X. Liao, Z. Liu, and S. Lan, "Complete chloroplast genome of Chloranthus henryi (chloranthaceae)," Mitochondrial DNA Part B, vol. 4, no. 2, pp. 2964-2965, 2019.

[23] X.-L. Yang, "Characterization of the complete chloroplast genome of Houttuynia cordata Thunb and phylogenetic relationships," Mitochondrial DNA Part B, vol. 5, no. 1, pp. 605606, 2020.

[24] M.-Q. Tang, S.-C. Yao, L.-X. Li, Z.-Z. Lan, and Z.-Z. Ling, "Establishment of AFLP Reaction System for Medicinal Plant Sarcandra glabra," Hubei Agricultural Sciences, vol. 17, 2012.

[25] Y. Xu, G.-Y. Wei, Y. Zhou, X.-F. Huang, and Y.-Q. Xu, “A set of novel microsatellite markers developed for a traditional Chinese medicinal plant, Sarcandra glabra (Chloranthaceae)," Journal of Genetics, vol. 94, no. S1, pp. 86-88, 2015.

[26] Y. Wei, Y. Chen, Y. Huang, J. Liu, and Y. Liang, "Molecular authentication and quantitative analysis of Sarcandra glabra and adulterated chloranthus products using SNP markers," Mitochondrial DNA Part A, vol. 27, no. 5, pp. 3618-3625, 2016.

[27] Anonymous, Sarcandra glabra, Flora of China, China, 1999.

[28] Q. Zhang, S. F. Taylor, and A. Antonelli, “Assessing the impact of phylogenetic incongruence on taxonomy, floral evolution, biogeographical history, and phylogenetic diversity," American Journal of Botany, vol. 102, pp. 1-15, 2015.

[29] M. A. R. Rashid, Y. Zhao, H. Zhang, J. Li, and Z. Li, "Nucleotide diversity, natural variation, and evolution of Flexible culm-1 and Strong culm-2 lodging resistance genes in rice," Genome, vol. 59, no. 7, pp. 473-483, 2016.

[30] R. V. Kantety, M. La Rota, D. E. Matthews, and M. E. Sorrells, "Data mining for simple sequence repeats in expressed sequence tags from barley, maize, rice, sorghum and wheat," Plant Molecular Biology, vol. 48, no. 5/6, pp. 501-510, 2002.

[31] H. Zhang, D. Zhang, M. Wang et al., "A core collection and mini core collection of Oryza sativa L. in China," Theoretical and Applied Genetics, vol. 122, no. 1, pp. 49-61, 2011.

[32] O. Thakur and G. S. Randhawa, "Identification and characterization of SSR, SNP and InDel molecular markers from RNASeq data of guar (Cyamopsis tetragonoloba, L. Taub.) roots," BMC Genomics, vol. 19, no. 1, p. 951, 2018.

[33] J. Xu, Y. Li, X. Ma et al., "Whole transcriptome analysis using next-generation sequencing of model species Setaria viridis to support C 4 photosynthesis research," Plant Molecular Biology, vol. 83, no. 1-2, pp. 77-87, 2013.

[34] S. Shah, C. Weinholdt, N. Jedrusik et al., "Whole-transcriptome analysis reveals genetic factors underlying flowering time regulation in rapeseed (Brassica napusL.)," Plant, Cell \& Environment, vol. 41, no. 8, pp. 1935-1947, 2018.

[35] R. C. Strange, P. W. Jones, and A. A. Fryer, "Glutathione Stransferase: genetics and role in toxicology," Toxicology Letters, vol. 112-113, pp. 357-363, 2000.

[36] P. J. Cock, C. J. Fields, N. Goto, M. L. Heuer, and P. M. Rice, "The Sanger FASTQ file format for sequences with quality scores, and the Solexa/Illumina FASTQ variants," Nucleic Acids Research, vol. 38, no. 6, pp. 1767-1771, 2010.

[37] M. G. Grabherr, B. J. Haas, M. Yassour et al., "Full-length transcriptome assembly from RNA-Seq data without a reference genome," Nature Biotechnology, vol. 29, no. 7, pp. 644-652, 2011.

[38] F. A. Simão, R. M. Waterhouse, P. Ioannidis, E. V. Kriventseva, and E. M. Zdobnov, "BUSCO: assessing genome assembly and annotation completeness with single-copy orthologs," Bioinformatics, vol. 31, no. 19, pp. 3210-3212, 2015. 
[39] B. Buchfink, C. Xie, and D. H. Huson, "Fast and sensitive protein alignment using DIAMOND," Nature Methods, vol. 12, no. 1, pp. 59-60, 2015.

[40] The UniProt Consortium, "UniProt: a hub for protein information," Nucleic Acids Research, vol. 43, no. D1, pp. D204D212, 2015.

[41] M. Kanehisa, M. Araki, S. Goto et al., "KEGG for linking genomes to life and the environment," Nucleic Acids Research, vol. 36, no. Database, pp. D480-D484, 2007.

[42] J. Huerta-Cepas, D. Szklarczyk, K. Forslund et al., "eggNOG 4.5: a hierarchical orthology framework with improved functional annotations for eukaryotic, prokaryotic and viral sequences," Nucleic Acids Research, vol. 44, no. D1, pp. D286-D293, 2016.

[43] M. Ashburner, C. A. Ball, J. A. Blake et al., "Gene ontology: tool for the unification of biology," Nature Genetics, vol. 25, no. 1, pp. 25-29, 2000.

[44] E. Camon, M. Magrane, D. Barrell et al., "The gene ontology annotation (goa) database: sharing knowledge in uniprot with gene ontology," Nucleic Acids Research, vol. 32, no. 90001, pp. 262D-2266, 2004.

[45] A. Untergasser, I. Cutcutache, T. Koressaar et al., "Primer3-new capabilities and interfaces," Nucleic Acids Research, vol. 40, no. 15, pp. e115-e115, 2012.

[46] A. Dobin, C. A. Davis, F. Schlesinger et al., "STAR: ultrafast universal RNA-seq aligner," Bioinformatics, vol. 29, no. 1, pp. 15-21, 2013.

[47] A. McKenna, M. Hanna, E. Banks et al., "The Genome Analysis Toolkit: a MapReduce framework for analyzing nextgeneration DNA sequencing data," Genome Research, vol. 20, no. 9, pp. 1297-1303, 2010.

[48] P. Cingolani, A. Platts, L. L. Wang et al., "A program for annotating and predicting the effects of single nucleotide polymorphisms, SnpEff: SNPs in the genome of Drosophila melanogaster strain w1118; iso-2; iso-3," Fly, vol. 6, no. 2, pp. 80-92, 2012.

[49] B. Langmead and S. L. Salzberg, "Fast gapped-read alignment with Bowtie 2," Nature Methods, vol. 9, no. 4, pp. 357-359, 2012.

[50] B. Li and C. N. Dewey, "RSEM: accurate transcript quantification from RNA-Seq data with or without a reference genome," BMC Bioinformatics, vol. 12, no. 1, p. 323, 2011. 